\title{
Three-Phase Freak Waves
}

\author{
Aleksandr O. SMIRNOV, Sergei G. MATVEENKO, Sergei K. SEMENOV \\ and Elena G. SEMENOVA \\ St.-Petersburg State University of Aerospace Instrumentation (SUAI), \\ 67 Bolshaya Morskaya Str., St.-Petersburg, 190000, Russia \\ E-mail: alsmir@guap.ru,MatveiS239@gmail.com,sksemenov@mail.ru,egsemenova@mail.ru
}

Received December 05, 2014, in final form April 11, 2015; Published online April 21, 2015

http://dx.doi.org/10.3842/SIGMA.2015.032

\begin{abstract}
In the article, we describe three-phase finite-gap solutions of the focusing nonlinear Schrödinger equation and Kadomtsev-Petviashvili and Hirota equations that exhibit the behavior of almost-periodic "freak waves". We also study the dependency of the solution parameters on the spectral curves.
\end{abstract}

Key words: nonlinear Schrödinger equation; Hirota equation; freak waves; theta function; reduction; covering; spectral curve

2010 Mathematics Subject Classification: 35Q55; 37C55

\section{Introduction}

This study was motivated by the intention to demonstrate the behavior of three-phase extreme waves. Most recent scientific research shows that the simplest and most universal model for such waves is the focusing nonlinear Schrödinger equation (NLS)

$$
i p_{t}+p_{x x}+2|p|^{2} p=0, \quad i^{2}=-1,
$$

Since 1968 the equation (1) has been describing distribution on the surface of the ocean of weakly nonlinear quasi-monochromatic wave packets with relatively steep fronts [44]. An application of this equation to the problems of nonlinear optics was known earlier [7]. Since the equation (1) is a model of first approximation, it appears in simulations of many weakly nonlinear phenomena. This equation has a wide range of applications ranging from plasma physics [28] to financial markets [43].

Among the properties of equation (1) there is a modulation instability that leads to the appearance of the so-called "freak waves" (in hydrodynamics known as "rogue waves") [2]. These waves represent amplitude peaks localized in space and time. In the last 20 years, first in hydrodynamics and then in nonlinear optics, these waves have been the object of numerous theoretical and experimental studies [3]. Such attention to the problem of the "freak waves" is due to the losses at oil platforms, tankers, container ships and other large vessels caused by the "rogue waves".

There are many more precise and more complex models, which give a more exact description of the "freak waves" [3]. These models can be divided into two classes. In the first class one can solve them analyticaly while in the second class one can use numerical methods only. Analytical methods include: inverse scattering transform method; finite-gap integration method; Bäcklund transform method; Darboux transform method; Hirota method.

In the present work, we use a finite-gap integration method. The works of Dubrovin, Novikov, Marchenko, Lax, McKean, van Moerbeke, Matveev, Its, Krichever [9, 10, 11, 12, 13, 21, 22, 26, 29, $31,33,35]$ give a description of this method (see also the review [32]). However, another method of constructing finite-gap solutions of integrable nonlinear equations exists [23, 24, 34, 36]. Let 
us remark that first method is based on Baker-Akhiezer function but the second one is based on some Fay's identities [14]. In our paper, we use the first method and Its and Kotlyarov's classic formulas $[18,20]$ (see also [6]).

Our goal here is to show the behavior of three-phase algebro-geometric solutions of NLS, KP-I and Hirota equations. Section 2 of this paper contains the basic notations and classic formulas for algebro-geometric solutions of integrable nonlinear equations under consideration. Section 3 is devoted to the periodicity of three-phase solutions of NLS, KP-I and Hirota equations. In Section 4 we consider an example of three-phase algebro-geometric solutions of KP-I and Hirota equations for different values of parameters.

\section{Finite-gap multi-phase solutions of the NLS equation}

The nonlinear differential equations that are integrated by methods of the algebraic geometry, can be obtained as a compatibility condition of the system of ordinary linear differential equations with a spectral parameter $[6,15,16]$. In particular, let us consider the following equations $[15,19,38]$

$$
Y_{x}=\mathfrak{U} Y, \quad Y_{z}=\mathfrak{V} Y, \quad Y_{t}=\mathfrak{W} Y,
$$

where

$$
\mathfrak{U}=-\lambda\left(\begin{array}{cc}
i & 0 \\
0 & -i
\end{array}\right)+\left(\begin{array}{cc}
0 & i \psi \\
-i \phi & 0
\end{array}\right), \quad \mathfrak{V}=2 \lambda \mathfrak{U}+\mathfrak{V}_{0}, \quad \mathfrak{W}=4 \lambda^{2} \mathfrak{U}+2 \lambda \mathfrak{W}_{0}+\mathfrak{W}_{1},
$$

$\lambda$ is a spectral parameter. Using these equations and additional relations

$$
\left(Y_{x}\right)_{z}=\left(Y_{z}\right)_{x}, \quad\left(Y_{x}\right)_{t}=\left(Y_{t}\right)_{x}
$$

one can easy obtain the so-called equations of zero curvature

$$
\mathfrak{U}_{z}-\mathfrak{V}_{x}+\mathfrak{U V}-\mathfrak{V} \mathfrak{U}=0 \quad \text { and } \quad \mathfrak{U}_{t}-\mathfrak{W}_{x}+\mathfrak{U} \mathfrak{W}-\mathfrak{W} \mathfrak{U}=0,
$$

which should be valid for all values of spectral parameter $\lambda$. Respectively, it follows from equations (3) that matrixes $\mathfrak{V}_{0}, \mathfrak{W}_{0}, \mathfrak{W}_{1}$ take the forms

$$
\mathfrak{W}_{0}=\mathfrak{V}_{0}=\left(\begin{array}{cc}
-i \psi \phi & -\psi_{x} \\
-\phi_{x} & i \psi \phi
\end{array}\right), \quad \mathfrak{W}_{1}=\left(\begin{array}{cc}
\psi_{x} \phi-\psi \phi_{x} & 2 i \psi^{2} \phi-i \psi_{x x} \\
-2 i \psi \phi^{2}+i \phi_{x x} & \psi \phi_{x}-\psi_{x} \phi
\end{array}\right),
$$

Also, $\mathfrak{W}=2 \lambda \mathfrak{V}+\mathfrak{W}_{1}$. Conditions (3) lead to additional system of equations (parities). The first system is the coupled nonlinear Schrödinger equation

$$
\begin{aligned}
& i \psi_{z}+\psi_{x x}-2 \psi^{2} \phi=0, \\
& i \phi_{z}-\phi_{x x}+2 \psi \phi^{2}=0,
\end{aligned}
$$

and the second system is the coupled modified Korteweg-de Vries equation

$$
\begin{aligned}
& \psi_{t}+\psi_{x x x}-6 \psi \phi \psi_{x}=0 \\
& \phi_{t}+\phi_{x x x}-6 \psi \phi \phi_{x}=0 .
\end{aligned}
$$

These two systems of the nonlinear differential equations are closely related to two other ones. Specifically, differentiating equations (4) with respect to $x$ and substituting them in (5), one obtains the coupled modified two-dimensional nonlinear Schrödinger equation in cone coordinates [27]

$$
\begin{aligned}
& i \psi_{t}+\psi_{x z}+2 i\left(\psi \phi_{x}-\phi \psi_{x}\right) \psi=0, \\
& i \phi_{t}-\phi_{x z}+2 i\left(\phi \psi_{x}-\psi \phi_{x}\right) \phi=0,
\end{aligned}
$$


Also, the functions $\psi(x, t,-\alpha t)$ and $\phi(x, t,-\alpha t)$ are solutions to the coupled integrable Hirota equation $(\alpha \in \mathbb{R})$

$$
\begin{aligned}
& i \psi_{t}+\psi_{x x}-2 \psi^{2} \phi-i \alpha\left(\psi_{x x x}-6 \psi \phi \psi_{x}\right)=0, \\
& i \phi_{t}-\phi_{x x}+2 \psi \phi^{2}-i \alpha\left(\phi_{x x x}-6 \psi \phi \phi_{x}\right)=0,
\end{aligned}
$$

if $\psi(x, z, t)$ and $\phi(x, z, t)$ are solutions of (4) and (5).

Systems of the nonlinear differential equations (4), (5) are the first two integrable systems from the AKNS hierarchy [15]. One of the features of finite-gap multi-phase solutions of the integrable nonlinear equations is that fact that in some sense they are the solutions of all hierarchy. Particulary, our solutions can be used for constructing solutions of generalized nonlinear Schrödinger equation [42]. By substituting $\phi= \pm \bar{\psi}$ into equation (4) we get a standard form of the nonlinear Schrödinger equation. Particularly, for $\phi=-\bar{\psi}$ equations (4) transform to $(1)[11,18,19]$ and equations (6) transform to the integrable Hirota equation $[4,8,17,30]$

$$
i \psi_{t}+\psi_{x x}+2|\psi|^{2} \psi-i \alpha\left(\psi_{x x x}+6|\psi|^{2} \psi_{x}\right)=0 .
$$

It is also easy to check that for any $\psi$ and $\phi$, that satisfy both (4) and (5) simultaneously, the function $u(x, z, t)=-2 \psi \phi$ is a solution of the Kadomtsev-Petviashvili-I equation (KP-I)

$$
3 u_{z z}=\left(4 u_{t}+u_{x x x}+6 u u_{x}\right)_{x} .
$$

In case $\phi= \pm \bar{\psi}$ this solution is a real function.

Finite-gap solutions of systems (4), (5) are parameterized by the hyperelliptic curve $\Gamma=$ $\{(\chi, \lambda)\}$ of the genus $g[15,38]$ :

$$
\Gamma: \chi^{2}=\prod_{j=1}^{2 g+2}\left(\lambda-\lambda_{j}\right)
$$

The branch points $\left(\lambda=\lambda_{j}, j=1, \ldots, 2 g+2\right)$ of this curve are the endpoints of the spectral arcs of continuous spectrum of Dirac operator (2). Infinitely far point of the spectrum corresponds two different points $\mathcal{P}_{\infty}^{ \pm}$on the curve $\Gamma$. In case $\phi=-\bar{\psi}$ the curve $\Gamma$ has the form

$$
\Gamma: \chi^{2}=\prod_{j=1}^{g+1}\left(\lambda-\lambda_{j}\right)\left(\lambda-\bar{\lambda}_{j}\right)=\lambda^{2 g+2}+\sum_{j=1}^{2 g+2} \chi_{j} \lambda^{2 g+2-j}, \quad \Im \chi_{j}=0, \quad \Im\left(\lambda_{j}\right) \neq 0 .
$$

Following a standard procedure of constructing finite-gap solutions $[6,11,38]$, for $\Gamma$ let us choose a canonical basis of cycles $\gamma^{t}=\left(a_{1}, \ldots, a_{g}, b_{1}, \ldots, b_{g}\right)$ with matrix of intersection indices

$$
C_{0}=\left(\begin{array}{cc}
0 & I \\
-I & 0
\end{array}\right)
$$

To satisfy the condition $\phi=-\bar{\psi}$, it is necessary $[6,11]$ that this basis of cycles is transformed according to the rules

$$
\widehat{\tau}_{1} \mathbf{a}=-\mathbf{a}, \quad \widehat{\tau}_{1} \mathbf{b}=\mathbf{b}+K \mathbf{a},
$$

where $\tau_{1}$ is anti-holomorphic involution, $\tau_{1}:(\chi, \lambda) \rightarrow(\bar{\chi}, \bar{\lambda})$.

Let us also consider normalized holomorphic differentials $d \mathcal{U}_{j}$ :

$$
\oint_{a_{k}} d \mathcal{U}_{j}=\delta_{k j}, \quad k, j=1, \ldots, g
$$


and a matrix of periods $B$ of the curve $\Gamma$ :

$$
B_{k j}=\oint_{b_{k}} d \mathcal{U}_{j}, \quad k, j=1, \ldots, g
$$

It is well known (see, for example, $[5,11]$ ) that the matrix $B$ is a symmetric matrix with positively defined imaginary part.

Let us introduce $g$-dimensional Riemann theta function with characteristics $\boldsymbol{\eta}, \boldsymbol{\zeta} \in \mathbb{R}^{g}[5,11$, 14]:

$$
\begin{aligned}
& \Theta\left[\boldsymbol{\eta}^{t} ; \boldsymbol{\zeta}^{t}\right](\mathbf{p} \mid B)=\sum_{\mathbf{m} \in \mathbb{Z}^{g}} \exp \left\{\pi i(\mathbf{m}+\boldsymbol{\eta})^{t} B(\mathbf{m}+\boldsymbol{\eta})+2 \pi i(\mathbf{m}+\boldsymbol{\eta})^{t}(\mathbf{p}+\boldsymbol{\zeta})\right\} \\
& \Theta\left[\mathbf{0}^{t} ; \mathbf{0}^{t}\right](\mathbf{p} \mid B) \equiv \Theta(\mathbf{p} \mid B)
\end{aligned}
$$

where $B$ is a matrix of periods, $\mathbf{p} \in \mathbb{C}^{g}$ and summation passes over an integer $g$-dimensional lattice.

Let us also define on $\Gamma$ normalized Abelian integrals of the second kind $\left(\Omega_{j}(\mathcal{P}), j=1,2,3\right)$ and the third kind $\left(\omega_{0}(\mathcal{P})\right)$ with the following asymptotic at infinitely distant points $\mathcal{P}_{\infty}^{ \pm}$:

$$
\begin{aligned}
& \oint_{a_{k}} d \Omega_{1}=\oint_{a_{k}} d \Omega_{2}=\oint_{a_{k}} d \Omega_{3}=\oint_{a_{k}} d \omega_{0}=0, \quad k=1, \ldots, g, \\
& \Omega_{1}(\mathcal{P})=\mp i\left(\lambda-K_{1}+O\left(\lambda^{-1}\right)\right), \quad \mathcal{P} \rightarrow \mathcal{P}_{\infty}^{ \pm}, \\
& \Omega_{2}(\mathcal{P})=\mp i\left(2 \lambda^{2}-K_{2}+O\left(\lambda^{-1}\right)\right), \quad \mathcal{P} \rightarrow \mathcal{P}_{\infty}^{ \pm}, \\
& \Omega_{3}(\mathcal{P})=\mp i\left(4 \lambda^{3}-K_{3}+O\left(\lambda^{-1}\right)\right), \quad \mathcal{P} \rightarrow \mathcal{P}_{\infty}^{ \pm}, \\
& \omega_{0}(\mathcal{P})=\mp\left(\ln \lambda-\ln K_{0}+O\left(\lambda^{-1}\right)\right), \quad \mathcal{P} \rightarrow \mathcal{P}_{\infty}^{ \pm}, \\
& \chi= \pm\left(\lambda^{g+1}+O\left(\lambda^{g}\right)\right), \quad \mathcal{P} \rightarrow \mathcal{P}_{\infty}^{ \pm} .
\end{aligned}
$$

Let us denote the vectors of $b$-periods of Abelian integrals of the second kind $\Omega_{1}(\mathcal{P}), \Omega_{2}(\mathcal{P})$, $\Omega_{3}(\mathcal{P})$ by $2 \pi i \mathbf{U}, 2 \pi i \mathbf{V}, 2 \pi i \mathbf{W}$ respectively.

Theorem 1 ([6, 38]). Function

$$
Y(\mathcal{P}, x, z, t)=\left(\begin{array}{ll}
y_{1}(\mathcal{P}, x, z, t) & y_{1}\left(\tau_{0} \mathcal{P}, x, z, t\right) \\
y_{2}(\mathcal{P}, x, z, t) & y_{2}\left(\tau_{0} \mathcal{P}, x, z, t\right)
\end{array}\right)
$$

where $\tau_{0}$ is hyperelliptic involution, $\tau_{0}:(\chi, \lambda) \rightarrow(-\chi, \lambda)$,

$$
\begin{aligned}
y_{1}(\mathcal{P}, x, z, t)= & \frac{\Theta(\mathcal{U}(\mathcal{P})+\mathbf{U} x+\mathbf{V} z+\mathbf{W} t-\mathbf{X}) \Theta(\mathbf{Z})}{\Theta(\mathcal{U}(\mathcal{P})-\mathbf{X}) \Theta(\mathbf{U} x+\mathbf{V} z+\mathbf{W} t+\mathbf{Z})} \\
& \times \exp \left\{\Omega_{1}(\mathcal{P}) x+\Omega_{2}(\mathcal{P}) z+\Omega_{3}(\mathcal{P}) t+i \Phi(x, z, t)\right\} \\
y_{2}(\mathcal{P}, x, z, t)= & \rho \frac{\Theta(\mathcal{U}(\mathcal{P})+\mathbf{U} x+\mathbf{V} z+\mathbf{W} t+\mathbf{\Delta}-\mathbf{X}) \Theta(\mathbf{Z}-\mathbf{\Delta})}{\Theta(\mathcal{U}(\mathcal{P})-\mathbf{X}) \Theta(\mathbf{U} x+\mathbf{V} z+\mathbf{W} t+\mathbf{Z})} \\
& \times \exp \left\{\Omega_{1}(\mathcal{P}) x+\Omega_{2}(\mathcal{P}) z+\Omega_{3}(\mathcal{P}) t-i \Phi(x, z, t)+\omega_{0}(\mathcal{P})\right\}
\end{aligned}
$$

is the eigenfunction of the Dirac operator (2) with functions

$$
\begin{aligned}
& \psi(x, z, t)=\frac{2 K_{0}}{\rho} \frac{\Theta(\mathbf{Z}) \Theta(\mathbf{U} x+\mathbf{V} z+\mathbf{W} t+\mathbf{Z}-\mathbf{\Delta})}{\Theta(\mathbf{Z}-\boldsymbol{\Delta}) \Theta(\mathbf{U} x+\mathbf{V} z+\mathbf{W} t+\mathbf{Z})} \exp \{2 i \Phi(x, z, t)\}, \\
& \phi(x, z, t)=2 \rho K_{0} \frac{\Theta(\mathbf{Z}-\boldsymbol{\Delta}) \Theta(\mathbf{U} x+\mathbf{V} z+\mathbf{W} t+\mathbf{Z}+\mathbf{\Delta})}{\Theta(\mathbf{Z}) \Theta(\mathbf{U} x+\mathbf{V} z+\mathbf{W} t+\mathbf{Z})} \exp \{-2 i \Phi(x, z, t)\},
\end{aligned}
$$


for any $z, t$ and $\rho \neq 0$. The functions (11) satisfy the equations (4) and (5). Here $\boldsymbol{\Delta}$ is the vector of holomorphic Abelian integrals, calculated along a path connecting $\mathcal{P}_{\infty}^{-}$and $\mathcal{P}_{\infty}^{+}$without crossing any of the basic cycles,

$$
\begin{aligned}
& \boldsymbol{\Delta}=\mathcal{U}\left(\mathcal{P}_{\infty}^{+}\right)-\mathcal{U}\left(\mathcal{P}_{\infty}^{-}\right), \quad \Phi(x, z, t)=K_{1} x+K_{2} z+K_{3} t \\
& \mathbf{X}=\mathcal{K}+\sum_{j=1}^{g} \mathcal{U}\left(\mathcal{P}_{j}\right), \quad \mathbf{Z}=\mathcal{U}\left(\mathcal{P}_{\infty}^{+}\right)-\mathbf{X}
\end{aligned}
$$

$\mathcal{K}$ is a vector of Riemann constants $[5,11,14,25] ; \mathcal{P}_{j}, j=1, \ldots, g$ is a non-special divisor. If the spectral curve $\Gamma$ satisfies the condition (9), then the following equalities hold

$$
\begin{aligned}
& |\psi|^{2}=-4 K_{0}^{2} \frac{\Theta(\mathbf{U} x+\mathbf{V} z+\mathbf{W} t+\mathbf{Z}-\mathbf{\Delta}) \Theta(\mathbf{U} x+\mathbf{V} z+\mathbf{W} t+\mathbf{Z}+\mathbf{\Delta})}{\Theta^{2}(\mathbf{U} x+\mathbf{V} z+\mathbf{W} t+\mathbf{Z})}, \\
& \Im \mathbf{U}=\Im \mathbf{V}=\Im \mathbf{W}=\Im \mathbf{Z}=\mathbf{0}, \quad K_{0}^{2}<0 .
\end{aligned}
$$

It is easy to see that the corresponding solution of KP-I equation (8) has the form

$$
u(x, z, t)=-8 K_{0}^{2} \frac{\Theta(\mathbf{U} x+\mathbf{V} z+\mathbf{W} t+\mathbf{Z}-\boldsymbol{\Delta}) \Theta(\mathbf{U} x+\mathbf{V} z+\mathbf{W} t+\mathbf{Z}+\boldsymbol{\Delta})}{\Theta^{2}(\mathbf{U} x+\mathbf{V} z+\mathbf{W} t+\mathbf{Z})},
$$

and that the square of amplitude of solution of Hirota equation (7) equals

$$
\left|\psi_{H}\right|^{2}(x, t)=-4 K_{0}^{2} \frac{\Theta(\mathbf{U} x+(\mathbf{V}-\alpha \mathbf{W}) t+\mathbf{Z}-\boldsymbol{\Delta}) \Theta(\mathbf{U} x+(\mathbf{V}-\alpha \mathbf{W}) t+\mathbf{Z}+\boldsymbol{\Delta})}{\Theta^{2}(\mathbf{U} x+(\mathbf{V}-\alpha \mathbf{W}) t+\mathbf{Z})} .
$$

\section{Features of three-phase solutions}

In case $g=3$, the basis of normalized holomorphic differentials is defined by the formula [6, 11]:

$$
d \mathcal{U}_{k}=\left(c_{k 1} \lambda^{2}+c_{k 2} \lambda+c_{k 3}\right) \frac{d \lambda}{\chi}
$$

where

$$
C=\left(A^{t}\right)^{-1}, \quad A_{j m}=\oint_{a_{j}} \lambda^{3-m} \frac{d \lambda}{\chi} .
$$

It follows from equation $(\ell$ is an arbitrary path on $\Gamma$ )

$$
\int_{\widehat{\tau} \ell} d \omega=\int_{\ell} \tau^{*} d \omega
$$

that

$$
\overline{A_{j m}}=\oint_{a_{j}} \overline{\lambda^{3-m} \frac{d \lambda}{\chi}}=\oint_{a_{j}} \tau_{1}^{*}\left(\lambda^{3-m} \frac{d \lambda}{\chi}\right)=\oint_{\widehat{\tau}_{1} a_{j}} \lambda^{3-m} \frac{d \lambda}{\chi}=-\oint_{a_{j}} \lambda^{3-m} \frac{d \lambda}{\chi}=-A_{j m} .
$$

Therefore $\bar{A}=-A$ and $\bar{C}=-C$. Similarly, with integrals on $b$-cycles, we obtain

$$
\bar{B}=-B-K \quad \text { or } \quad \Re B=-\frac{1}{2} K .
$$

It follows from bilinear relations of Riemann (see, for example, $[5,6,11]$ ) that the coordinates of the vectors $\mathbf{U}, \mathbf{V}, \mathbf{W}$ can be written as

$$
U_{m}=-i\left(\left.\frac{d \mathcal{U}_{m}}{d \xi_{-}}\right|_{\xi_{-}=0}-\left.\frac{d \mathcal{U}_{m}}{d \xi_{+}}\right|_{\xi_{+}=0}\right), \quad V_{m}=-2 i\left(\left.\frac{d^{2} \mathcal{U}_{m}}{d \xi_{-}^{2}}\right|_{\xi_{-}=0}-\left.\frac{d^{2} \mathcal{U}_{m}}{d \xi_{+}^{2}}\right|_{\xi_{+}=0}\right)
$$




$$
W_{m}=-2 i\left(\left.\frac{d^{3} \mathcal{U}_{m}}{d \xi_{-}^{3}}\right|_{\xi_{-}=0}-\left.\frac{d^{3} \mathcal{U}_{m}}{d \xi_{+}^{3}}\right|_{\xi_{+}=0}\right)
$$

where $\xi_{ \pm}=1 / \lambda$ are local parameters in the neighborhood of infinitely distant points $\mathcal{P}_{\infty}^{ \pm}$. Calculating the derivatives we obtain the relations

$$
U_{m}=-2 i c_{m 1}, \quad V_{m}=2 i \chi_{1} c_{m 1}-4 i c_{m 2}, \quad W_{m}=i\left(4 \chi_{2}-3 \chi_{1}^{2}\right) c_{m 1}+4 i \chi_{1} c_{m 2}-8 i c_{m 3},
$$

or

$$
(\mathbf{U}, \mathbf{V}, \mathbf{W})=i C\left(\begin{array}{ccc}
-2 & 2 \chi_{1} & 4 \chi_{2}-3 \chi_{1}^{2} \\
0 & -4 & 4 \chi_{1} \\
0 & 0 & -8
\end{array}\right)
$$

It follows from (14) that the vectors $\mathbf{U}, \mathbf{V}, \mathbf{W}$ are real and linearly independent. Therefore, $\mathbf{U}, \mathbf{V}, \mathbf{W}$ are the basis vectors in $\mathbb{R}^{3}$. Hence, any vector from $\mathbb{R}^{3}$ can be presented in the form of the linear combinations of these vectors. In particular, for the vectors of the periods of the three-dimensional theta-functions $\mathbf{e}_{1}^{t}=(1,0,0), \mathbf{e}_{2}^{t}=(0,1,0), \mathbf{e}_{3}^{t}=(0,0,1)$ we can write the following relations

$$
\mathbf{e}_{k}=\mathcal{X}_{k} \mathbf{U}+\mathcal{Z}_{k} \mathbf{V}+\mathcal{T}_{k} \mathbf{W}
$$

Therefore, three-phase solutions (13) of equation KP-I (8) are the periodic functions in a threedimensional space

$$
u\left(x+\mathcal{X}_{k}, z+\mathcal{Z}_{k}, t+\mathcal{T}_{k}\right)=u(x, z, t) .
$$

If a three-phase solution of (8) has a form of freak waves, then the maxima of its amplitude are located in nodes of a three-dimensional lattice with edges $\left(\mathcal{X}_{k}, \mathcal{Z}_{k}, \mathcal{T}_{k}\right)$. These edges can be found by an inversion of the matrix $(\mathbf{U}, \mathbf{V}, \mathbf{W})$ :

$$
\left(\begin{array}{ccc}
\mathcal{X}_{1} & \mathcal{X}_{2} & \mathcal{X}_{3} \\
\mathcal{Z}_{1} & \mathcal{Z}_{2} & \mathcal{Z}_{3} \\
\mathcal{T}_{1} & \mathcal{T}_{2} & \mathcal{T}_{3}
\end{array}\right)=(\mathbf{U}, \mathbf{V}, \mathbf{W})^{-1}=i\left(\begin{array}{ccc}
1 / 2 & \chi_{1} / 4 & \chi_{2} / 4-\chi_{1}^{2} / 16 \\
0 & 1 / 4 & \chi_{1} / 8 \\
0 & 0 & 1 / 8
\end{array}\right) A^{t}
$$

Therefore, for three-phases solutions of equation KP-I (8) it is possible to describe their behavior in the following way: after a time interval $\Delta t=\mathcal{T}_{k}$ a surface of solution $u(x, z)$ reproduces itself with a shift on the $X O Z$ plane by the $\left(\mathcal{X}_{k}, \mathcal{Z}_{k}\right)$ vector.

As the three-phase solution of the equations (1) depends on two coordinates, $x$ and $z$, and the third coordinate $t$ is considered to be a parameter, the value of amplitude of this solution depends on the distance between the nodes of the given three-dimensional lattice and a plane $t=t_{0}$. Hence, in contrast to the case of the two-phase solution [39, 40,41], where the change of initial phase $\mathbf{Z}$ led to trivial shift of the solution on $X O Z$ plane, the amplitude of the three-phase solution (11) of equations (1) depends on a choice of initial phase $\mathbf{W} t_{0}+\mathbf{Z}$ in a slightly more complicated fashion.

\section{An example of three-phase solution}

Let us consider a spectral curve $\Gamma_{3}=\{\chi, \lambda\}$ of genus $g=3$ :

$$
\Gamma_{3}: \chi^{2}=\left(\left(\lambda-\lambda_{0}\right)^{4}-2 a^{2}\left(\lambda-\lambda_{0}\right)^{2} \cos 2 \varphi+a^{4}\right)\left(\left(\lambda-\lambda_{0}\right)^{4}-2 b^{2}\left(\lambda-\lambda_{0}\right)^{2} \cos 2 \varphi+b^{4}\right),
$$

where $0<a<b, \pi / 4<\varphi<\pi / 2$. 


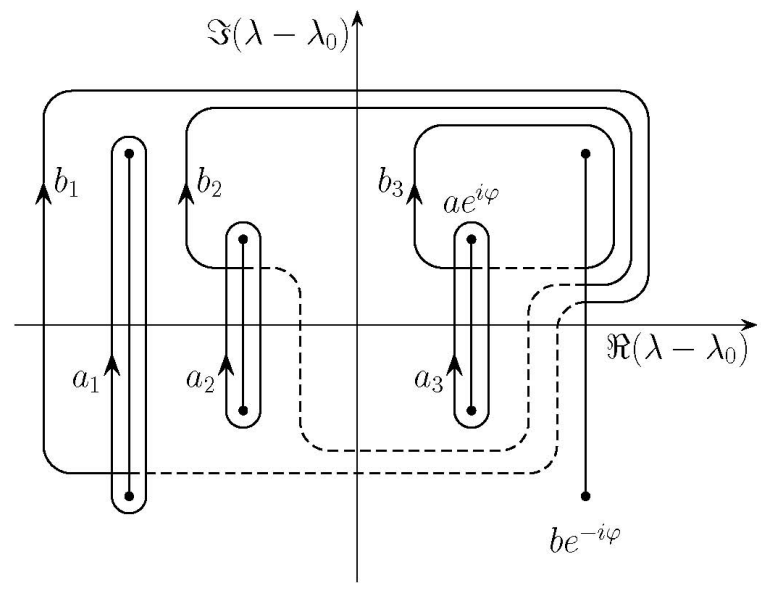

Figure 1. Canonical basis of cycles on $\Gamma_{3}$.

Let us choose the basis of cycles on $\Gamma_{3}$ as it is shown on Fig. 1.

It is easy to check that the anti-holomorphic involution $\tau_{1}$ transforms the canonical basis of cycles using the rule (10) with the matrix

$$
K=\left(\begin{array}{lll}
0 & 1 & 1 \\
1 & 0 & 1 \\
1 & 1 & 0
\end{array}\right)
$$

There are also three holomorphic involutions on $\Gamma_{3}$ :

$$
\begin{aligned}
& \tau_{0}:(\chi, \lambda) \rightarrow(-\chi, \lambda) \\
& \tau_{2}:(\chi, \lambda) \rightarrow\left(\chi, 2 \lambda_{0}-\lambda\right), \\
& \tau_{3}:(\chi, \lambda) \rightarrow\left(a^{2} b^{2}\left(\lambda-\lambda_{0}\right)^{-4} \chi, \lambda_{0}+a b\left(\lambda-\lambda_{0}\right)^{-1}\right) .
\end{aligned}
$$

As a corollary, the curve $\Gamma_{3}$ covers the following two curves:

1) $\Gamma_{1}=\Gamma_{3} / \tau_{2}$ of genus $g=1$

$$
\Gamma_{1}: \chi_{+}^{2}=\left(t^{2}-2 a^{2} t \cos 2 \varphi+a^{4}\right)\left(t^{2}-2 b^{2} t \cos 2 \varphi+b^{4}\right),
$$

2) $\Gamma_{2}=\Gamma_{3} /\left(\tau_{0} \tau_{2}\right)$ of genus $g=2$

$$
\Gamma_{2}: \chi_{-}^{2}=t\left(t^{2}-2 a^{2} t \cos 2 \varphi+a^{4}\right)\left(t^{2}-2 b^{2} t \cos 2 \varphi+b^{4}\right),
$$

where $t=\left(\lambda-\lambda_{0}\right)^{2}, \chi_{+}=\chi, \chi_{-}=\left(\lambda-\lambda_{0}\right) \chi$, and

$$
\frac{d t}{\chi_{+}}=\frac{2\left(\lambda-\lambda_{0}\right) d \lambda}{\chi}, \quad \frac{t d t}{\chi_{-}}=\frac{2\left(\lambda-\lambda_{0}\right)^{2} d \lambda}{\chi}, \quad \frac{d t}{\chi_{-}}=\frac{2 d \lambda}{\chi} .
$$

The curves $\Gamma_{1}$ and $\Gamma_{2}$ are shown on Figs. 2 and 3 , where $t_{1}=b^{2} e^{2 i \varphi}, t_{2}=a^{2} e^{2 i \varphi}$.

The coverings generate the following transformations of cycles:

$$
\left(\begin{array}{l}
a_{1} \\
a_{2} \\
a_{3}
\end{array}\right) \rightarrow S\left(\begin{array}{l}
a^{1} \\
a_{1}^{2} \\
a_{2}^{2}
\end{array}\right)+P\left(\begin{array}{l}
b^{1} \\
b_{1}^{2} \\
b_{2}^{2}
\end{array}\right), \quad\left(\begin{array}{l}
b_{1} \\
b_{2} \\
b_{3}
\end{array}\right) \rightarrow Q\left(\begin{array}{l}
a^{1} \\
a_{1}^{2} \\
a_{2}^{2}
\end{array}\right)+R\left(\begin{array}{l}
b^{1} \\
b_{1}^{2} \\
b_{2}^{2}
\end{array}\right)
$$




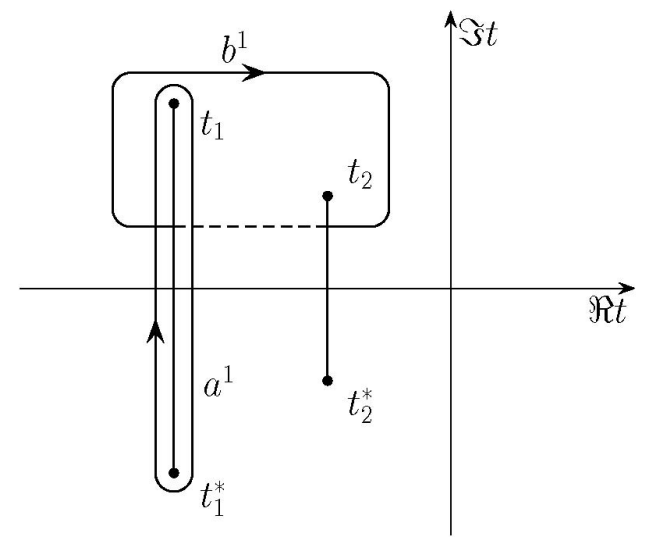

Figure 2. The curve $\Gamma_{1}$.

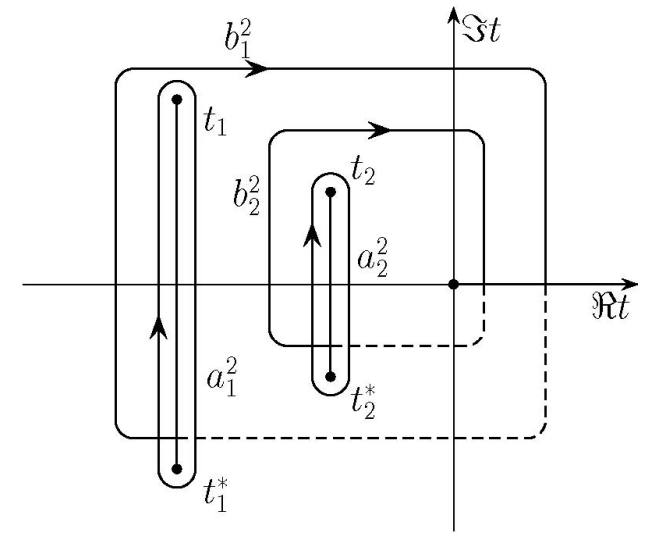

Figure 3. The curve $\Gamma_{2}$.

where

$$
\begin{aligned}
S=\left(\begin{array}{ccc}
-1 & 1 & 0 \\
1 & 0 & -1 \\
1 & 0 & 1
\end{array}\right), & P=\left(\begin{array}{ccc}
0 & -2 & 0 \\
0 & 0 & 2 \\
0 & 0 & -2
\end{array}\right) \\
Q=\left(\begin{array}{ccc}
-1 & 1 & 0 \\
0 & 0 & -1 \\
0 & 0 & 1
\end{array}\right), & R=\left(\begin{array}{ccc}
0 & 0 & 0 \\
1 & 1 & 1 \\
1 & 1 & -1
\end{array}\right) .
\end{aligned}
$$

Recall that these matrices should satisfy the relations

$$
S^{t} Q=Q^{t} S, \quad R^{t} P=P^{t} R, \quad S^{t} R-Q^{t} P=n I
$$

where $I$ is identity matrix, $n=2$ is the number of covering sheets.

Due to involution $\tau_{3}$, the curve $\Gamma_{2}$ covers two elliptic curves $\Gamma_{ \pm}$(Figs. 4 and 5 ):

$$
\Gamma_{ \pm}: \nu_{ \pm}^{2}=(s \pm 2 a b)\left(s^{2}-2\left(a^{2}+b^{2}\right) s \cos 2 \varphi+a^{4}+b^{4}+2 a^{2} b^{2} \cos 4 \varphi\right)
$$

where

$$
s=t+\frac{a^{2} b^{2}}{t}, \quad \nu_{ \pm}=\frac{t \pm a b}{t^{2}} \chi_{-}, \quad \frac{d s}{\nu_{ \pm}}=\frac{(t \mp a b) d t}{\chi_{-}} .
$$

The coverings of $\Gamma_{2}$ on $\Gamma_{ \pm}$generate the following cycles mappings

$$
\left(\begin{array}{l}
a_{1}^{2} \\
a_{2}^{2}
\end{array}\right) \rightarrow\left(\begin{array}{cc}
1 & 1 \\
-1 & 1
\end{array}\right)\left(\begin{array}{l}
a_{+} \\
a_{-}
\end{array}\right), \quad\left(\begin{array}{l}
b_{1}^{2} \\
b_{2}^{2}
\end{array}\right) \rightarrow\left(\begin{array}{cc}
1 & 1 \\
-1 & 1
\end{array}\right)\left(\begin{array}{l}
b_{+} \\
b_{-}
\end{array}\right)
$$

As a result, we have

$$
\begin{aligned}
& \left(\begin{array}{l}
a_{1} \\
a_{2} \\
a_{3}
\end{array}\right) \rightarrow\left(\begin{array}{ccc}
-1 & 1 & 1 \\
1 & 1 & -1 \\
1 & -1 & 1
\end{array}\right)\left(\begin{array}{l}
a^{1} \\
a_{+} \\
a_{-}
\end{array}\right)+\left(\begin{array}{ccc}
0 & -2 & -2 \\
0 & -2 & 2 \\
0 & 2 & -2
\end{array}\right)\left(\begin{array}{l}
b_{1} \\
b_{+} \\
b_{-}
\end{array}\right), \\
& \left(\begin{array}{l}
b_{1} \\
b_{2} \\
b_{3}
\end{array}\right) \rightarrow\left(\begin{array}{ccc}
-1 & 1 & 1 \\
0 & 1 & -1 \\
0 & -1 & 1
\end{array}\right)\left(\begin{array}{l}
a^{1} \\
a_{+} \\
a_{-}
\end{array}\right)+\left(\begin{array}{lll}
0 & 0 & 0 \\
1 & 0 & 2 \\
1 & 2 & 0
\end{array}\right)\left(\begin{array}{l}
b_{1} \\
b_{+} \\
b_{-}
\end{array}\right)
\end{aligned}
$$




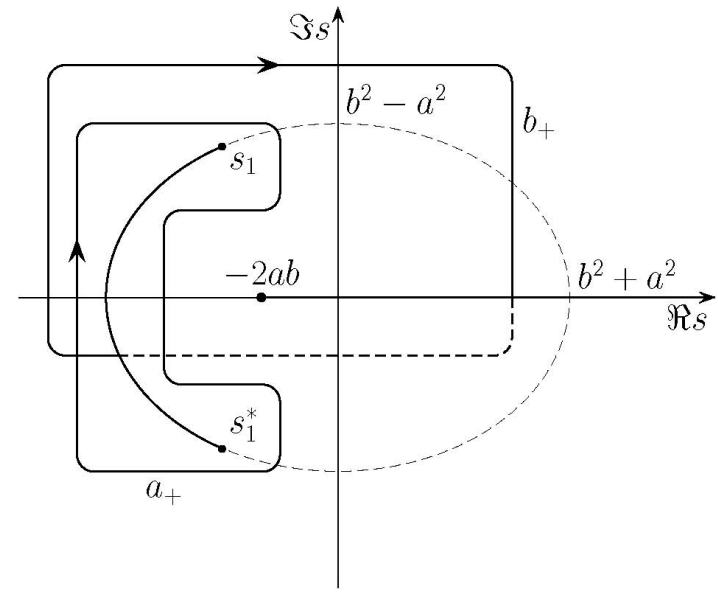

Figure 4. The curve $\Gamma_{+}$.

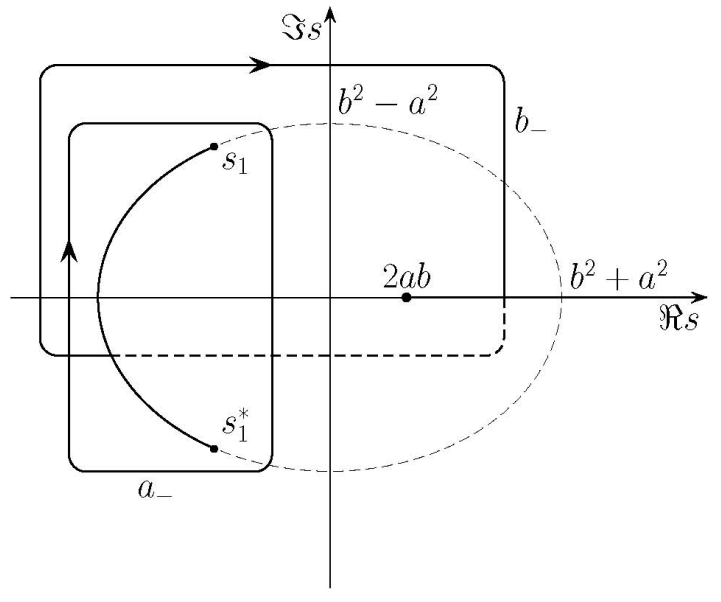

Figure 5. The curve $\Gamma_{-}$.

From (15), (16) and relations

$$
\begin{aligned}
& \frac{d \lambda}{\chi}=\frac{1}{4 a b} \frac{d s}{\nu_{-}}-\frac{1}{4 a b} \frac{d s}{\nu_{+}}, \quad \frac{\lambda d \lambda}{\chi}=\frac{1}{2} \frac{d t}{\chi_{+}}+\frac{\lambda_{0}}{4 a b} \frac{d s}{\nu_{-}}-\frac{\lambda_{0}}{4 a b} \frac{d s}{\nu_{+}}, \\
& \frac{\lambda^{2} d \lambda}{\chi}=\lambda_{0} \frac{d t}{\chi_{+}}+\frac{\lambda_{0}^{2}+a b}{4 a b} \frac{d s}{\nu_{-}}-\frac{\lambda_{0}^{2}-a b}{4 a b} \frac{d s}{\nu_{+}}
\end{aligned}
$$

it follows that

$$
\begin{aligned}
C= & \left(\begin{array}{ccc}
\mathfrak{c}_{1}+\mathfrak{c}_{3} & -2 \lambda_{0}\left(\mathfrak{c}_{1}+\mathfrak{c}_{3}\right) & \left(\lambda_{0}^{2}-a b\right) \mathfrak{c}_{1}+\left(\lambda_{0}^{2}+a b\right) \mathfrak{c}_{3} \\
\mathfrak{c}_{1} & \mathfrak{c}_{2}-2 \lambda_{0} \mathfrak{c}_{1} & \left(\lambda_{0}^{2}-a b\right) \mathfrak{c}_{1}-\lambda_{0} \mathfrak{c}_{2} \\
\mathfrak{c}_{3} & \mathfrak{c}_{2}-2 \lambda_{0} \mathfrak{c}_{3} & \left(\lambda_{0}^{2}+a b\right) \mathfrak{c}_{3}-\lambda_{0} \mathfrak{c}_{2}
\end{array}\right), \\
B= & \left(\begin{array}{ccc}
i\left(\mathfrak{b}_{1}+\mathfrak{b}_{3}\right) & i \mathfrak{b}_{1}-1 / 2 & i \mathfrak{b}_{3}-1 / 2 \\
i \mathfrak{b}_{1}-1 / 2 & i\left(\mathfrak{b}_{1}+\mathfrak{b}_{2}\right) & i \mathfrak{b}_{2}-1 / 2 \\
i \mathfrak{b}_{3}-1 / 2 & i \mathfrak{b}_{2}-1 / 2 & i\left(\mathfrak{b}_{2}+\mathfrak{b}_{3}\right)
\end{array}\right),
\end{aligned}
$$

where

$$
\begin{array}{rlrl}
\mathfrak{c}_{1} & =\frac{1}{2\left(\alpha_{1}-2 \beta_{1}\right)}, & \mathfrak{c}_{2}=\frac{1}{2 \alpha_{2}}, & \mathfrak{c}_{3}=\frac{1}{2\left(\alpha_{3}-2 \beta_{3}\right)}, \\
i \mathfrak{b}_{1}=\frac{\alpha_{1}}{2\left(\alpha_{1}-2 \beta_{1}\right)}, & i \mathfrak{b}_{2}=\frac{\beta_{2}}{2 \alpha_{2}}, & i \mathfrak{b}_{3}=\frac{\alpha_{3}}{2\left(\alpha_{3}-2 \beta_{3}\right)}, \\
\alpha_{1}=\frac{1}{2} \oint_{a_{+}} \frac{d s}{\nu_{+}}, & \alpha_{2}=\frac{1}{2} \oint_{a^{1}} \frac{d t}{\chi_{+}}, & \alpha_{3}=\frac{1}{2} \oint_{a_{-}} \frac{d s}{\nu_{-}} \\
\beta_{1}=\frac{1}{2} \oint_{b_{+}} \frac{d s}{\nu_{+}}, & \beta_{2}=\frac{1}{2} \oint_{b^{1}} \frac{d t}{\chi_{+}}, & \beta_{3}=\frac{1}{2} \oint_{b_{-}} \frac{d s}{\nu_{-}} .
\end{array}
$$

From the structure of the matrix $B$ and from the matrix version of Appel's theorem [37] it follows that the Riemann theta function of curve $\Gamma_{3}$ equals:

$$
\begin{aligned}
\Theta(\mathbf{p} \mid B)= & f\left(\widetilde{p}_{1}, \widetilde{p}_{2}, \widetilde{p}_{3}\right)=\vartheta_{3}\left(\widetilde{p}_{1} \mid h_{1}\right) \vartheta_{3}\left(\widetilde{p}_{2} \mid h_{2}\right) \vartheta_{3}\left(\widetilde{p}_{3} \mid h_{3}\right)+\vartheta_{4}\left(\widetilde{p}_{1} \mid h_{1}\right) \vartheta_{1}\left(\widetilde{p}_{2} \mid h_{2}\right) \vartheta_{1}\left(\widetilde{p}_{3} \mid h_{3}\right) \\
& +\vartheta_{1}\left(\widetilde{p}_{1} \mid h_{1}\right) \vartheta_{4}\left(\widetilde{p}_{2} \mid h_{2}\right) \vartheta_{1}\left(\widetilde{p}_{3} \mid h_{3}\right)+\vartheta_{1}\left(\widetilde{p}_{1} \mid h_{1}\right) \vartheta_{1}\left(\widetilde{p}_{2} \mid h_{2}\right) \vartheta_{4}\left(\widetilde{p}_{3} \mid h_{3}\right),
\end{aligned}
$$

where $\widetilde{p}_{j}=p_{j}+p_{j+1}-p_{j+2}, p_{j+3} \equiv p_{j}, h_{j}=\exp \left(-4 \mathfrak{b}_{j}\right)$. 


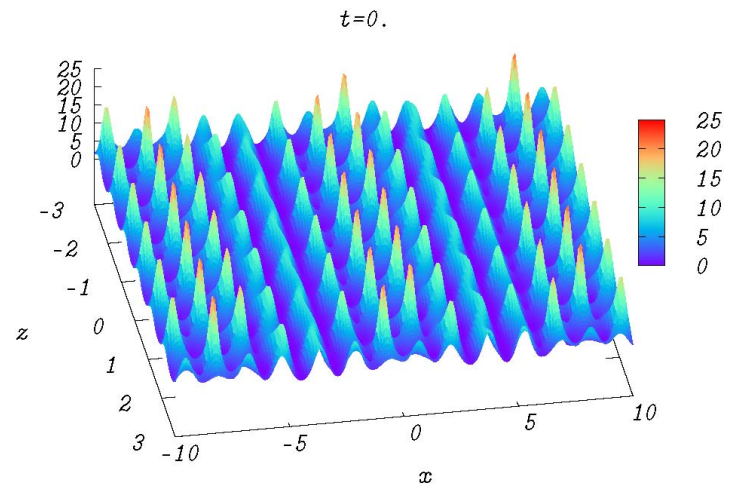

Figure 6. Three-phase solution of KP-I equation for $\lambda_{0}=0, t=0$.

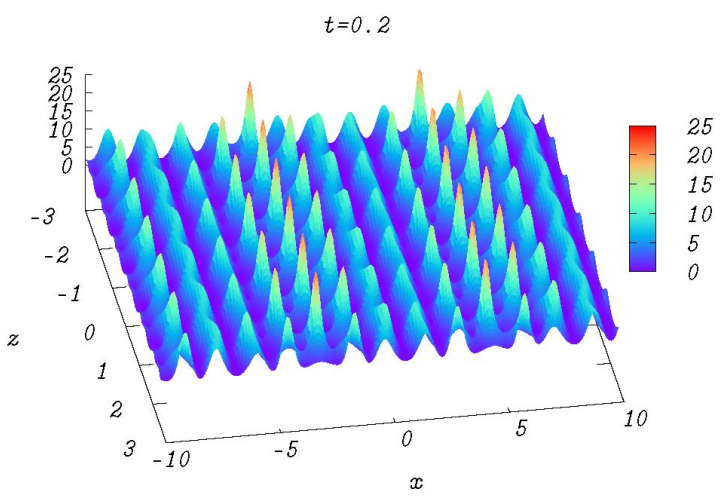

Figure 8. Three-phase solution of KP-I equation for $\lambda_{0}=0, t=0.2$.

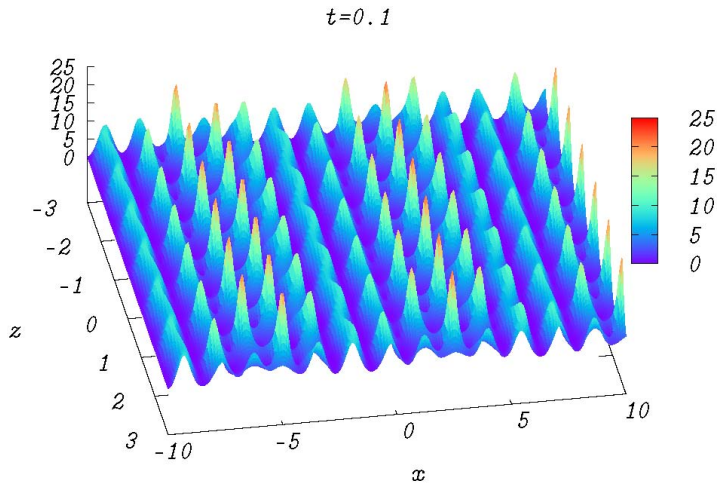

Figure 7. Three-phase solution of KP-I equation for $\lambda_{0}=0, t=0.1$.

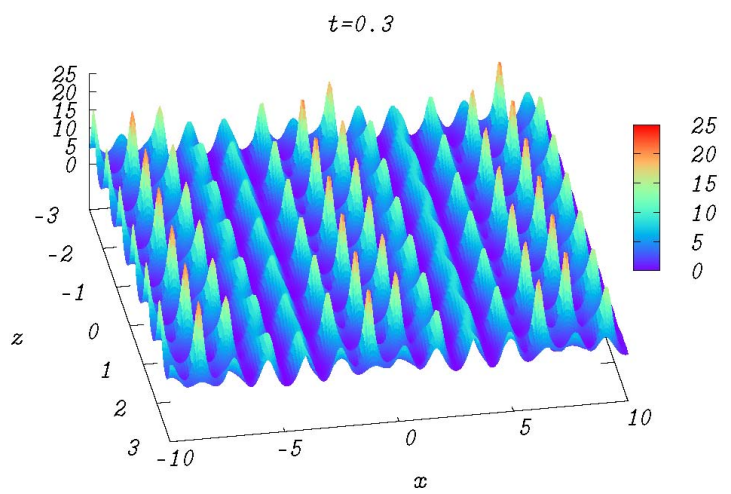

Figure 9. Three-phase solution of KP-I equation for $\lambda_{0}=0, t=0.3$.

The functions $\vartheta_{j}(p \mid h)$ are Jacoby elliptic theta functions [1]:

$$
\begin{aligned}
& \vartheta_{1}(p \mid h)=2 \sum_{m=1}^{\infty}(-1)^{m-1} h^{(m-1 / 2)^{2}} \sin [(2 m-1) \pi p] \\
& \vartheta_{2}(p \mid h)=2 \sum_{m=1}^{\infty} h^{(m-1 / 2)^{2}} \cos [(2 m-1) \pi p] \\
& \vartheta_{3}(p \mid h)=1+2 \sum_{m=1}^{\infty} h^{m^{2}} \cos (2 m \pi p) \\
& \vartheta_{4}(p \mid h)=1+2 \sum_{m=1}^{\infty}(-1)^{m} h^{m^{2}} \cos (2 m \pi p)
\end{aligned}
$$

Using the reduced form of theta function (17) and values for the vectors of periods, one obtains the following formula for a squared absolute value of the three-phase solution (12) of the focusing NLS equation (1)

$$
\begin{aligned}
|\psi|^{2}= & -4 K_{0}^{2} f\left(k_{1} x+\kappa_{1} t+\delta_{1}, k_{2} z+\delta_{2}, k_{3} x+\kappa_{3} t+\delta_{3}\right) \\
& \times f\left(k_{1} x+\kappa_{1} t-\delta_{1}, k_{2} z-\delta_{2}, k_{3} x+\kappa_{3} t-\delta_{3}\right) \times\left\{f\left(k_{1} x+\kappa_{1} t, k_{2} z, k_{3} x+\kappa_{3} t\right)\right\}^{-2},
\end{aligned}
$$




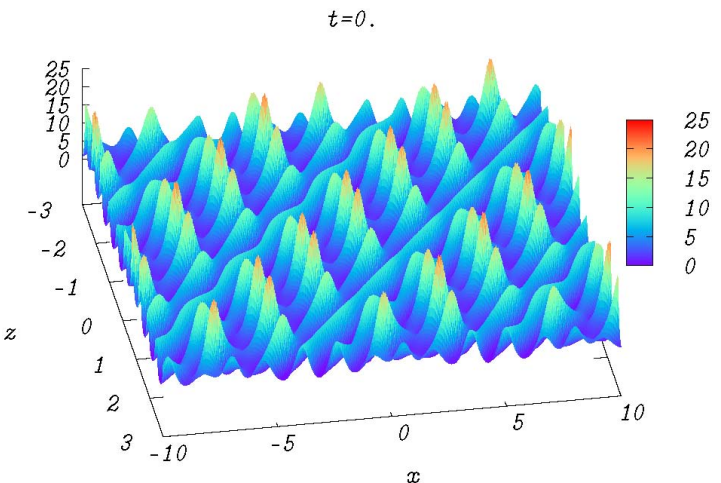

Figure 10. Three-phase solution of KP-I equation for $\lambda_{0}=k_{2} /\left(4 k_{1}\right), t=0$.

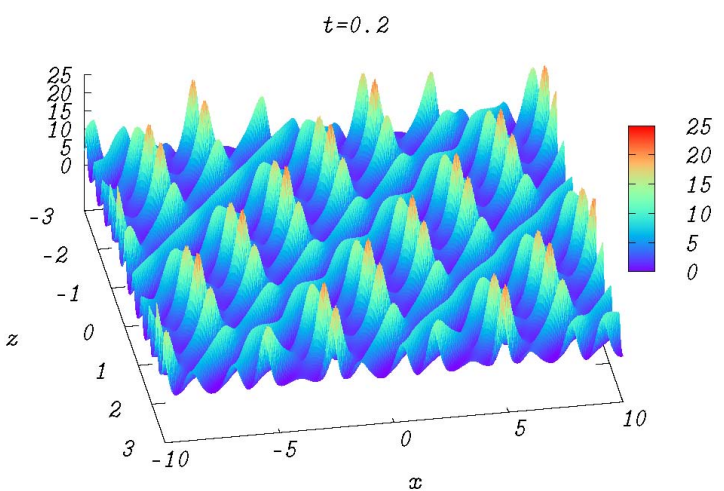

Figure 12. Three-phase solution of KP-I equation for $\lambda_{0}=k_{2} /\left(4 k_{1}\right), t=0.2$.

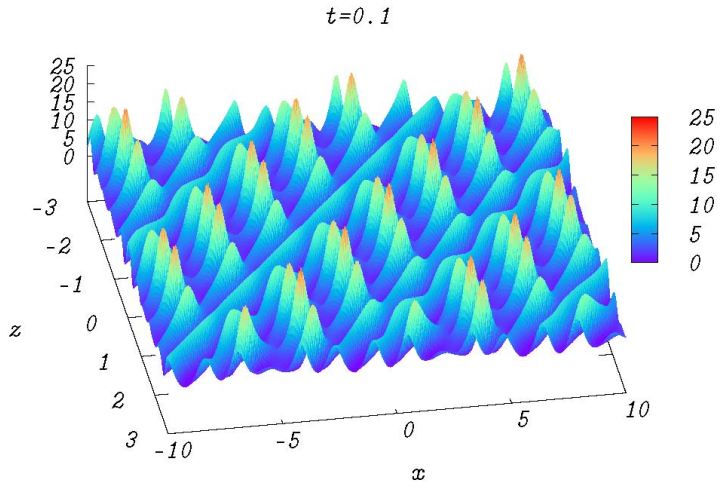

Figure 11. Three-phase solution of KP-I equation for $\lambda_{0}=k_{2} /\left(4 k_{1}\right), t=0.1$.

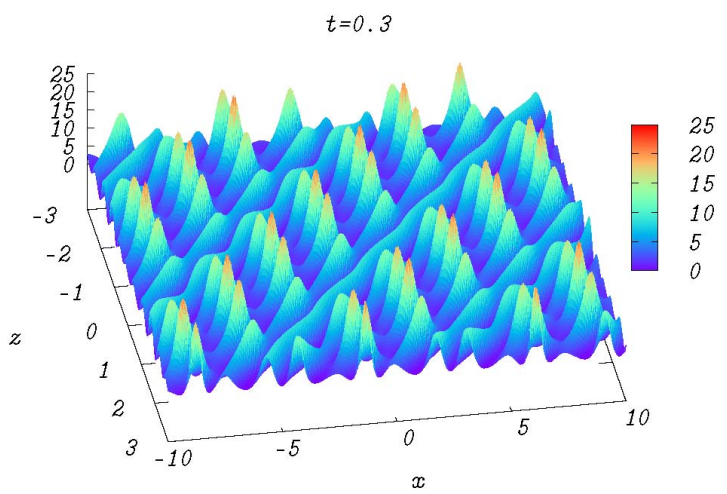

Figure 13. Three-phase solution of KP-I equation for $\lambda_{0}=k_{2} /\left(4 k_{1}\right), t=0.3$.

where the function $f\left(\widetilde{p}_{1}, \widetilde{p}_{2}, \widetilde{p}_{3}\right)$ is defined by equation (17), and

$$
\begin{aligned}
& k_{1}=-4 i \mathfrak{c}_{1}, \quad k_{2}=-8 i \mathfrak{c}_{2}, \quad k_{3}=-4 i \mathfrak{c}_{3}, \\
& \kappa_{1}=4 k_{1}\left(3 \lambda_{0}^{2}-a b+\left(a^{2}+b^{2}\right) \cos (2 \varphi)\right), \quad \kappa_{3}=4 k_{3}\left(3 \lambda_{0}^{2}+a b+\left(a^{2}+b^{2}\right) \cos (2 \varphi)\right) .
\end{aligned}
$$

(17), (18) imply that for $\lambda_{0}=0$ the amplitude of the constructed solution of NLS equation (1) is a periodic function of $z$, and for $\lambda_{0}=0, \varphi=\frac{1}{2} \arccos \left(\frac{ \pm a b}{a^{2}+b^{2}}\right)$ it is a periodic function of $z$ and $t$.

Recall that the three-phase solution $u(x, z, t)$ of the KP-I equation (8) and the square of amplitude of three-phase solution of Hirota equation $(7),\left|\psi_{\mathrm{H}}(x, t)\right|^{2}$, can be constructed from (18) by using relations $u(x, z, t)=2|\psi(x, z, t)|^{2}$ and $\left|\psi_{\mathrm{H}}(x, t)\right|^{2}=|\psi(x, t,-\alpha t)|^{2}$.

The three-phase solution of KP-I equation for $a b=1, \sqrt{b / a}=1.3, \varphi=0.3 \pi$ at the different moment of time $t$ and for $\lambda_{0}=0$ is presented on the Figs. 6-9. One can see the same solution for $\lambda_{0}=k_{2} /\left(4 k_{1}\right)$ on the Figs. 10-13. It is easy to see all three phases of solution on Figs. 6-13. Two phases are shortwaves and the third phase is a long-wave envelope. One can see also on Figs. 6-9 that the solution for $\lambda_{0}=0$ is periodic in $z$, and that the long-wave envelope moves to the right side.

The three-phase solution of Hirota equation for $a b=1, \sqrt{b / a}=1.3, \varphi=0.3 \pi, \alpha=0.1$ and for different values of $\lambda_{0}$ is presented in Figs. 14-17. It is easy to see all three phases of solution only in Fig. 15. 


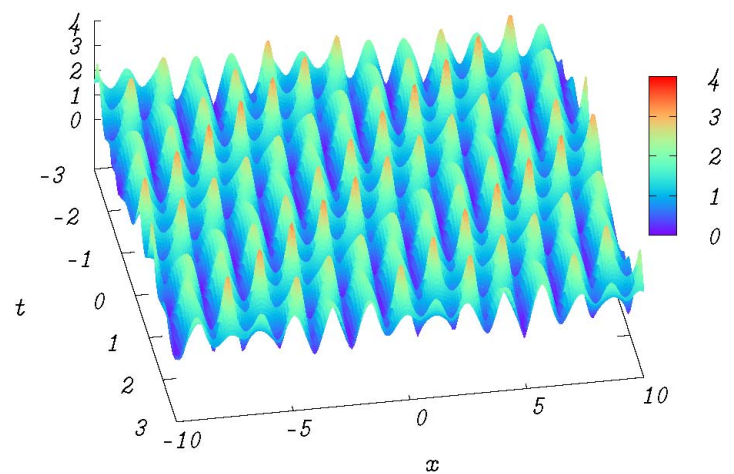

Figure 14. Amplitude of three-phase solution of Hirota equation for $\lambda_{0}=0$.

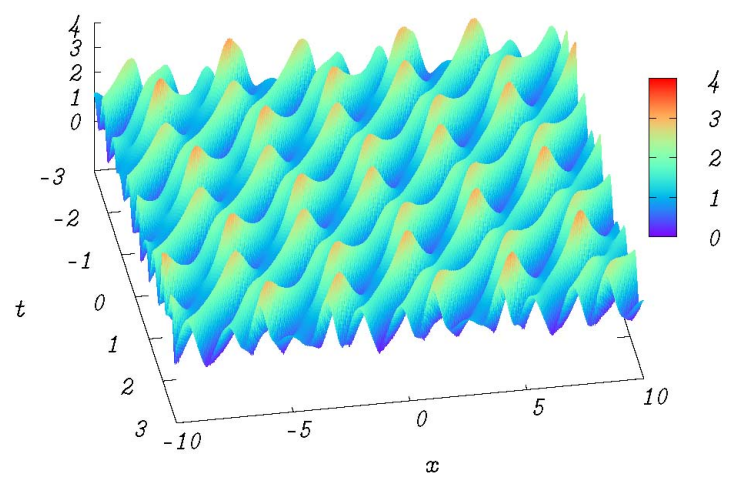

Figure 16. Amplitude of three-phase solution of Hirota equation for $\lambda_{0}=k_{2} /\left(4 k_{1}\right)$.

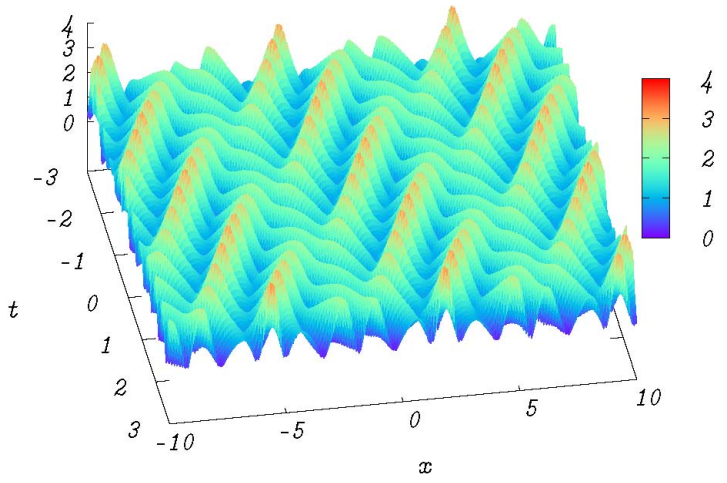

Figure 15. Amplitude of three-phase solution of Hirota equation for $\lambda_{0}=4$.

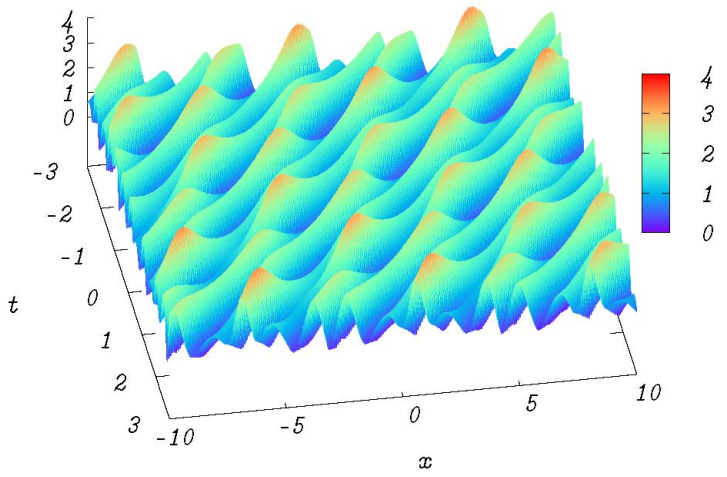

Figure 17. Amplitude of three-phase solution of Hirota equation for $\lambda_{0}=k_{2} /\left(4 k_{3}\right)$.

\section{Acknowledgements}

Authors thank Professor V.B. Matveev for his support and the discussions that we held over this paper and quasi-rational solutions of the NLS equation. This research was conducted within the framework of the State order of the Ministry of Education and Science of Russian Federation, and partially supported by RFBR (research project 14-01-00589_a).

\section{References}

[1] Akhiezer N.I., Elements of the theory of elliptic functions, Translations of Mathematical Monographs, Vol. 79, Amer. Math. Soc., Providence, RI, 1990.

[2] Akhmediev N.N., Ankiewicz A., Solitons, nonlinear pulses and beams, Chapman \& Hall, London, 1997.

[3] Akhmediev N., Pelinovsky E. (Editors), Discussion \& debate: rogue waves - towards a unifying concept?, Eur. Phys. J. Special Topics 185 (2010), 266 pages.

[4] Ankiewicz A., Soto-Crespo J.M., Akhmediev N., Rogue waves and rational solutions of the Hirota equation, Phys. Rev. E 81 (2010), 046602, 8 pages.

[5] Baker H.F., Abel's theorem and the allied theory including the theory of theta functions, Cambridge University Press, Cambridge, 1897.

[6] Belokolos E.D., Bobenko A.I., Enol'skii V.Z., Its A.R., Matveev V.B., Algebro-geometric approach to nonlinear integrable equations, Springer Series in Nonlinear Dynamics, Springer-Verlag, Berlin, 1994. 
[7] Chiao R.Y., Garmire E., Townes C.H., Self-trapping of optical beams, Phys. Rev. Lett. 13 (1964), 479-482.

[8] Dai C.Q., Zhang J.F., New solitons for the Hirota equation and generalized higher-order nonlinear Schrödinger equation with variable coefficients, J. Phys. A: Math. Gen. 39 (2006), 723-737.

[9] Dubrovin B.A., Inverse problem for periodic finite-zoned potentials in the theory of scattering, Funct. Anal. Appl. 9 (1975), 61-62.

[10] Dubrovin B.A., Periodic problems for the Korteweg-de Vries equation in the class of finite band potentials, Funct. Anal. Appl. 9 (1975), 215-223.

[11] Dubrovin B.A., Theta functions and non-linear equations, Russian Math. Surveys 36 (1981), no. 2, 11-92.

[12] Dubrovin B.A., Matveev V.B., Novikov S.P., Nonlinear equations of Korteweg-de Vries type, finite-band linear operators and Abelian varieties, Russian Math. Surveys 31 (1976), no. 1, 59-146.

[13] Dubrovin B.A., Novikov S.P., A periodicity problem for the Korteweg-de Vries and Sturm-Liouville equations. Their connection with algebraic geometry, Sov. Math. Dokl. 15 (1974), 1597-1601.

[14] Fay J.D., Theta functions on Riemann surfaces, Lecture Notes in Math., Vol. 352, Springer-Verlag, Berlin New York, 1973.

[15] Gesztesy F., Holden H., Soliton equations and their algebro-geometric solutions. Vol. I. (1+1)-dimensional continuous models, Cambridge Studies in Advanced Mathematics, Vol. 79, Cambridge University Press, Cambridge, 2003.

[16] Gesztesy F., Holden H., Michor J., Teschl G., Soliton equations and their algebro-geometric solutions. Vol. II. (1 + 1)-dimensional discrete models, Cambridge Studies in Advanced Mathematics, Vol. 114, Cambridge University Press, Cambridge, 2008.

[17] Guo B., Ling L., Liu Q.P., Nonlinear Schrödinger equation: generalized Darboux transformation and rogue wave solutions, Phys. Rev. E 85 (2012), 026607, 9 pages, arXiv:1108.2867.

[18] Its A.R., Inversion of hyperelliptic integrals, and integration of nonlinear differential equations, Vestnik Leningrad. Univ. (1976), no. 7, 39-46.

[19] Its A.R., "Isomonodromy" solutions of equations of zero curvature, Math. USSR-Izv. 26 (1986), 497-529.

[20] Its A.R., Kotlyarov V.P., On a class of solutions of the nonlinear Schrödinger equation, Dokl. Akad. Nauk USSR Ser. A 11 (1976), 965-968.

[21] Its A.R., Matveev V.B., Hill's operator with finitely many gaps, Funct. Anal. Appl. 9 (1975), 65-66.

[22] Its A.R., Matveev V.B., Schrödinger operators with the finite-band spectrum and $N$-soliton solutions of the Korteweg-de Vries equation, Theoret. and Math. Phys. 23 (1975), 343-355.

[23] Kalla C., Klein C., New construction of algebro-geometric solutions to the Camassa-Holm equation and their numerical evaluation, Proc. R. Soc. Lond. Ser. A 468 (2012), 1371-1390, arXiv:1109.5301.

[24] Kalla C., Klein C., On the numerical evaluation of algebro-geometric solutions to integrable equations, Nonlinearity 25 (2012), 569-596, arXiv:1107.2108.

[25] Krazer A., Lehrbuch der Thetafunktionen, Teubner, Leipzig, 1903.

[26] Krichever I.M., Methods of algebraic geometry in the theory of non-linear equations, Russian Math. Surveys 32 (1977), no. 6, 185-213.

[27] Kundu A., Mukherjee A., Naskar T., Modelling rogue waves through exact dynamical lump soliton controlled by ocean currents, Proc. R. Soc. Lond. Ser. A 470 (2014), 20130576, 20 pages, arXiv:1204.0916.

[28] Kuznetsov E.A., Solitons in a parametrically unstable plasma, Sov. Phys. Dokl. 22 (1977), 507-508.

[29] Lax P.D., Periodic solutions of the KdV equations, in Nonlinear Wave Motion (Proc. AMS-SIAM Summer Sem., Clarkson Coll. Tech., Potsdam, N.Y., 1972), Lectures in Appl. Math., Vol. 15, Amer. Math. Soc., Providence, R.I., 1974, 85-96.

[30] Li C.Z., He J.S., Darboux transformation and positons of the inhomogeneous Hirota and the Maxwell-Bloch equation, Sci. China Phys. Mech. Astronomy 57 (2014), 898-907, arXiv:1210.2501.

[31] Marchenko V.A., The periodic Korteweg-de Vries problem, Math. USSR Sb. 24 (1974), 319-344.

[32] Matveev V.B., 30 years of finite-gap integration theory, Philos. Trans. R. Soc. Lond. Ser. A 366 (2008), 837-875.

[33] McKean H.P., van Moerbeke P., The spectrum of Hill's equation, Invent. Math. 30 (1975), $217-274$.

[34] Mumford D., Tata lectures on theta. II. Jacobian theta functions and differential equations, Progress in Mathematics, Vol. 43, Birkhäuser Boston, Inc., Boston, MA, 1984. 
[35] Novikov S.P., The periodic problem for the Korteweg-de Vries equation, Funct. Anal. Appl. 8 (1974), $236-246$.

[36] Previato E., Hyperelliptic quasiperiodic and soliton solutions of the nonlinear Schrödinger equation, Duke Math. J. 52 (1985), 329-377.

[37] Smirnov A.O., A matrix analogue of a theorem of Appell and reductions of multidimensional Riemann theta-functions, Math. USSR Sb. 61 (1988), 379-388.

[38] Smirnov A.O., Elliptic solutions of the nonlinear Schrödinger equation and a modified Korteweg-de Vries equation, Mat. Sb. 185 (1994), 103-114.

[39] Smirnov A.O., Solution of a nonlinear Schrödinger equation in the form of two-phase freak waves, Theoret. and Math. Phys. 173 (2012), 1403-1416.

[40] Smirnov A.O., Periodic two-phase "rogue waves", Math. Notes 94 (2013), 897-907.

[41] Smirnov A.O., Semenova E.G., Zinger V., Zinger N., On a periodic solution of the focusing nonlinear Schrödinger equation, arXiv:1407.7974.

[42] Wang L.H., Porsezian K., He J.S., Breather and rogue wave solutions of a generalized nonlinear Schrödinger equation, Phys. Rev. E 87 (2013), 053202, 10 pages, arXiv:1304.8085.

[43] Yan Z., Vector financial rogue waves, Phys. Lett. A 375 (2011), 4274-4279, arXiv:1101.3107.

[44] Zakharov V.E., Stability of periodic waves of finite amplitude on the surface of a deep fluid, J. Appl. Mech. Tech. Phys. 9 (1968), 190-194. 